\title{
Time Scavengers: An Educational Website to Communicate Climate Change and Evolutionary Theory to the Public through Blogs, Web Pages, and Social Media Platforms
}

\author{
Adriane R. Lam, Jennifer E. Bauer², Susanna Fraass ${ }^{3}$, Sarah Sheffield ${ }^{4}$, Maggie R. Limbeck ${ }^{5}$, Rose M. Borden ${ }^{5}$, \\ Megan E. Thompson-Munson ${ }^{6}$, Andrew J. Fraass ${ }^{7}$, J. Michael Hils ${ }^{8}$, Cameron E. Muskelly ${ }^{9}$, Kyle R. Hartshorn ${ }^{10}$, and \\ Raquel Bryant ${ }^{1}$
}

${ }^{1}$ University of Massachusetts Amherst; ${ }^{2}$ University of Florida; ${ }^{3}$ Bristol UK; ${ }^{4}$ University of South Florida; ${ }^{5}$ University of Tennessee Knoxville; ${ }^{6}$ University of Wyoming; ${ }^{7}$ University of Bristol; ${ }^{8}$ University of Dayton; ${ }^{9}$ Paleontology Association of Georgia; ${ }^{10}$ Dry Dredgers

Keywords: Science Communication, Evolution, Climate Change, Digital Classroom

Publication Date: February 25, 2019

DOI: https://doi.org/10.15695/jstem/v2i1.05

\begin{abstract}
Climate change and evolution are topics at the forefront of political discussions, debates, and the public sphere. Regardless of evidence on both topics, the public as a whole still believes they are under debate. It is imperative that the public have access to correct and easy-to-digest information on these topics to make informed environmental and ecological decisions. To date, scientifically accurate digital platforms aimed at informing the public on these topics are overly complex and jargon-ridden. Time Scavengers (www.timescavengers.blog) was created to address these issues and is maintained by a group of academics, graduate students, avocational scientists, and educators. The site includes many informational pages about geology, climate change, and evolution, all written for the public with useful descriptions and figures. To make the process of science more transparent, the site includes blogs to provide insight into data collection and interpretation, field work, and public outreach. The site also includes additional pages with links to relevant content and activities appropriate for K-12 classrooms. The overarching goals of the site are to bridge the gap between scientists and the public through engaging, informational pages and personal experiences and to increase science literacy through easy-to-digest content.
\end{abstract}

\section{INTRODUCTION}

The world is in an age of communication and information-sharing via social media platforms (e.g., Facebook, Twitter, Instagram) and digital archives (blogs, web sites, news articles, informal articles, etc.). These digital spaces, or social media ecosystems, are a means of sharing information rapidly and from which active users are influenced by the opinions, experiences, and ideas of others (Hanna et al., 2011). It is no surprise that scientists have taken to blogging and social media posting to explain their research and reach a broader audience, including colleagues, policy makers, journalists, and the public. Some highly debated issues in the public sphere are climate change and evolution; thus, these topics are often blogged, posted, or written about by scientists, science communicators, and journalists. Frequently, digital media that attempt to explain these two topics are filled with information that is too complex, jargon-ridden, or overly simplified to the extent that scientific meaning is lost.
This is problematic, as few Americans attain an advanced degree in a geoscience field and fully understand the concepts behind long-term climate change and evolutionary theory compared to the total population. In 2016 alone, just 1440 out of over 323 million Americans earned a $\mathrm{PhD}$ in a geological sciences field (including geochemistry, mineralogy, geomorphology, paleontology, stratigraphy, glacial geology; National Center for Science and Engineering Statistics, 2016; United States Census Bureau, 2016). In addition to complex information, sites that share disinformation (false information that is intentionally spread) and misinformation (false or misleading information; Lazer et al., 2018) on these two topics are rampant.

In addition to the aforementioned problems, there is a lack of digital media wholly and thoroughly explaining where climate and evolution data come from and how the information is interpreted, as well as provide an insider view 
of the scientists working with the data. Although there are several science and personal blogs run by scientists who talk about their science, they often do so in a general way without delving into details about their procedures, data, or how they interpret their results. Although science blogs have great potential in disseminating information, they typically provide the readers with the expertise and knowledge from only a single viewpoint (Wilkins, 2008). In addition, science blogs tend to not reach their realized potential to disseminate studies, data, and offer an avenue for the public to post thoughtful questions. There remains a dearth of blogs in which scientists have thoughtful, meaningful conversations related to scientific discoveries, theories, or controversial topics with the public.

To address the above issues related to science blogs and easy-to-digest information, we developed a new website with informational pages and blogs (Figure 1), called Time Scavengers (www.timescavengers.blog). Time Scavengers is a digital space for the public to learn about general science and foundational geologic principles, with the main focus

Figure 1. Current site structure and web map of Time Scavengers. The static pages include the informational content of the website and additional resources that we aggregated for users. The blog pages are updated at least once a month and are frequently promoted through social media.

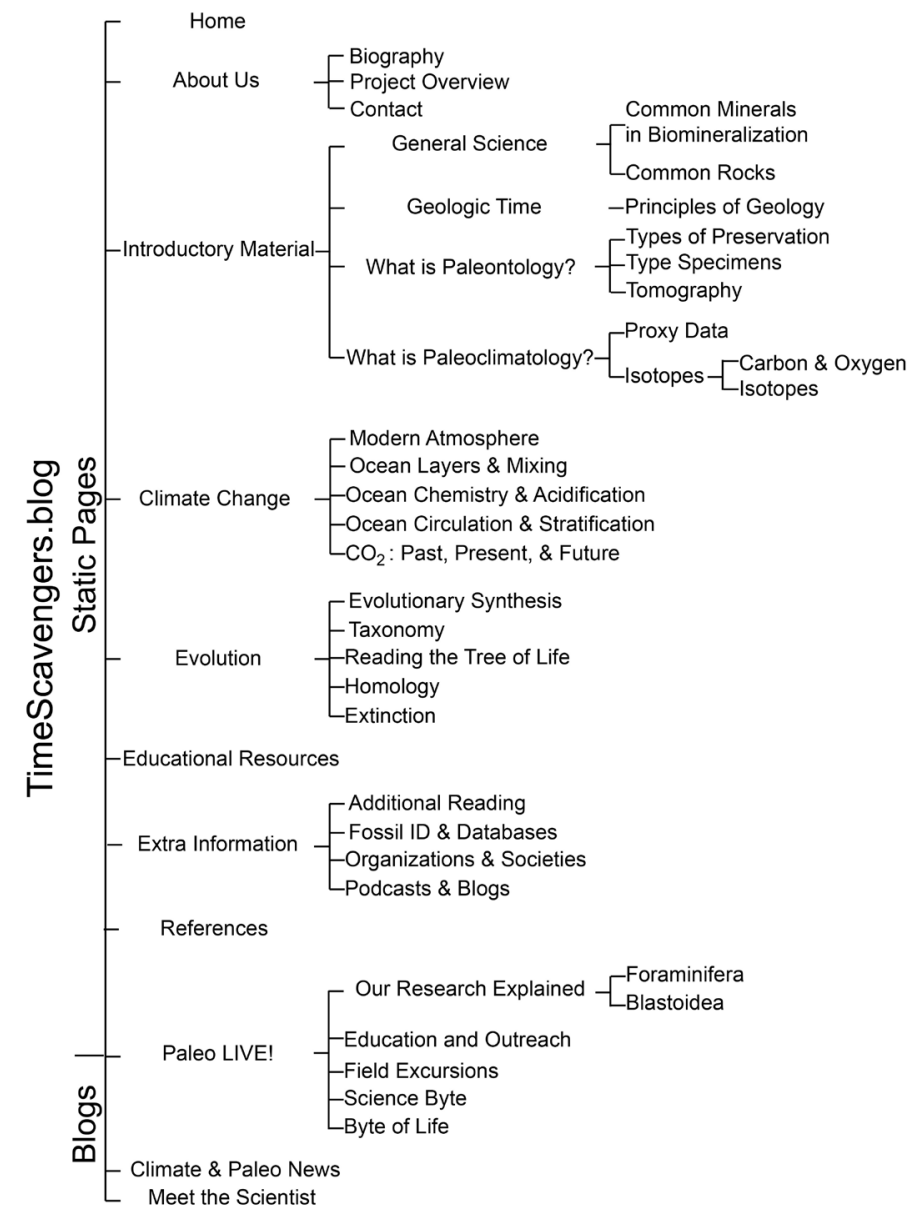

of teaching the scientific evidence and underlying theories of climate change and evolution. The site is written for the general public and structured for both people who are new to the disciplines and those with a general understanding of the topics. The site contains six blog components, each with the purpose of explaining scientific findings, showcasing the work of avocational and professional scientists, and introducing the public to the lives of scientists and the research they conduct.

The site content, pages, and collaborator posts are managed and maintained by two curators (ARL and JEB) who are geoscientists specializing in paleoceanography, paleontology, and science communication. Currently, ten site collaborators who range in geoscience careers from faculty to avocational paleontologists and stratigraphers contribute to Time Scavengers' blog components to share their experiences in the field, participating in education and outreach activities, conducting scientific research, and teaching. One of our collaborators is also our chief editor (SF), who aside from the two curators, is the primary editor of the content and ensures the pages and blog content is free of scientific jargon and reader-friendly. Site collaborators come from diverse socioeconomic, cultural, social identifies, and scientific backgrounds, with a wide range of research interests represented. Herein lies the strength of the site collaborators: they bring their experiences, expertise, and perspectives about science to the forefront of geoscience education and outreach. The multiple collaborator viewpoints and experiences provide a direct connection to a broad community (i.e., the general public), as voices to which non-traditional scientists and the public can relate. The presence of several collaborators from different career stages and backgrounds helps the public connect to the Time Scavengers platform through shared experiences (Hanna et al., 2011). Together, the Time Scavengers team has built a digital classroom experience to teach anyone interested in science about climate change, evolution, and the people who produce the science at the forefront of these two topics.

\section{SITE HOSTING AND MANAGEMENT}

The Time Scavengers website is currently hosted through WordPress.com with a Business plan. This plan is the most economical (\$300/year) that allows Google Analytics to run in the background of the site to collect data and statistics on site visitors. These data are being used to assess best practices related to running an educational website. ARL and JEB discussed the idea to develop Time Scavengers during December 2016 and spent six months outlining the structure of the site, writing informational pages, creating figures appropriate for our intended audience, writing initial blog posts, and recruiting collaborators. Weekly, JEB and ARL spent approximately 10-20 hours building site content. 
The site was publicly announced to our social media communities on July 6, 2017. To elevate site reach, we shared the Time Scavengers URL with the Women in Paleontology, Paleoceanography, Tennessee Science Teachers Association, and Paleontology Education Facebook groups. Initially, the site curators shared new blog posts released on the site on their personal Facebook pages. As our audience grew, a Facebook page specifically for Time Scavengers was created and released on October 9, 2017. In January 2018, six months after the site's public release, ARL and JEB spent time reassessing how frequently we were releasing posts and analyzed site visitor data from Google Analytics. We then began posting two blogs a week to our social media platforms and an additional post each Friday featuring a fossil species. From January 2018 onward, regularly scheduled posts increased the number of site visitors, and we began to reach a more global audience from different age groups (Figure 2).

To reach a broader audience, we created both Twitter and Instagram accounts to share posts and information. Our Twitter account was created on July 6, 2017 and our Instagram account went live on September 11, 2018 (Figure 2C).
Initially, the links to the newly released blog posts were copied onto a Twitter and Facebook post with some explanatory text. This did not look as eye-catching and professional as other related educational sites' social media posts (e.g., The FOSSIL Project), so on September 19, 2018, we began making social media posts using Canva.com, a free graphic design platform.

Because Time Scavengers includes a large number of blogs and site collaborators, it requires a rigorous management system. All files and information related to the site are kept online through Google Drive. Here, every collaborator has their own folder where they write their blog posts and contribute to collaborative posts. Collaborators are required to write one post a month, thus dedicating only 1-3 hours per month to Time Scavengers. Only the site curators and editor, ARL, JEB, and SF, have administrator privileges on WordPress. Once posts are finalized in the Google Drive folder, either ARL or JEB upload them to the site for subsequent editing. Currently, two new blog posts are released weekly. To manage the release date of the posts, we employ Hoostsuite.com, a free (with available paid upgrades) social media management tool that allows us to upload our Canva

Figure 2. Data from Google Analytics collected on Time Scavengers visitors, July 6, 2017-December 17, 2018. (A) Location of site visitors. People are being reached across the world, with the largest number of visitors from the U.S. (B) Age of visitors to the site, with a heavy bias towards the 18-34 age range. (C) Number of site visitors per day since Time Scavengers' public release. Note change in axis scale between July and October 2017. Peaks in the data indicate when posts are released. Major changes to the site and social media tools added are noted on the timeline in red arrows.
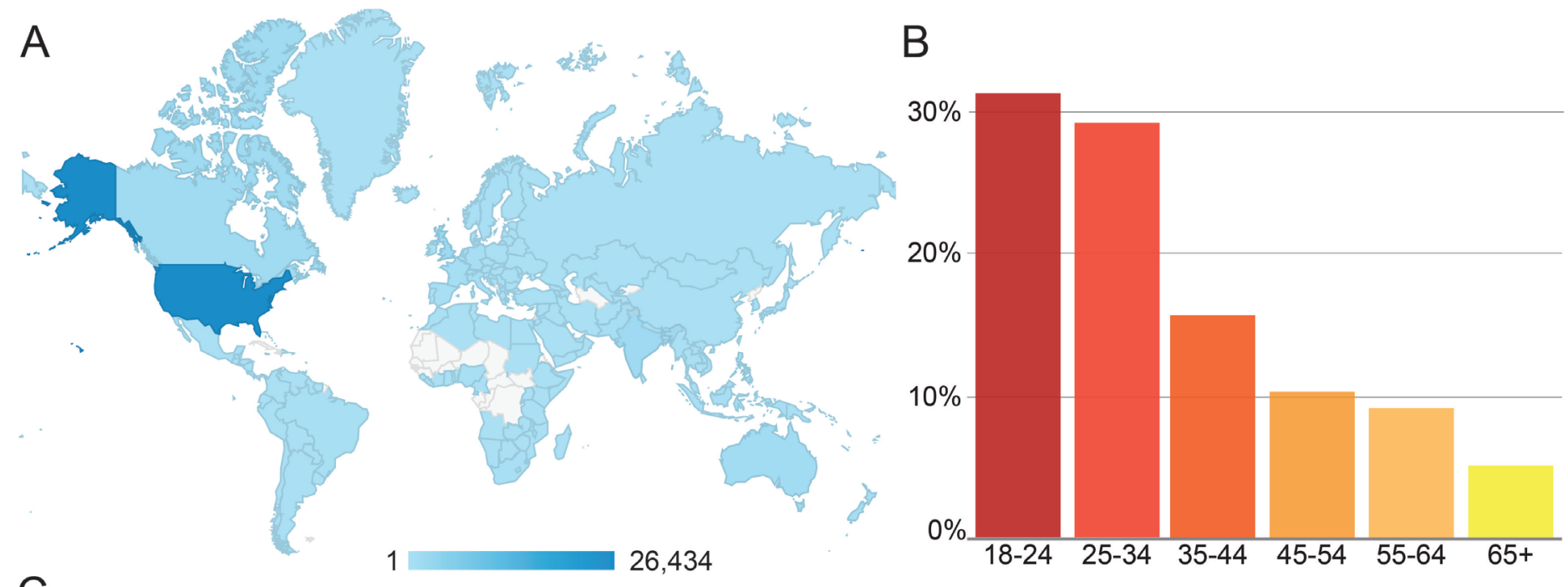

\section{C}

Site and Twitter

account release

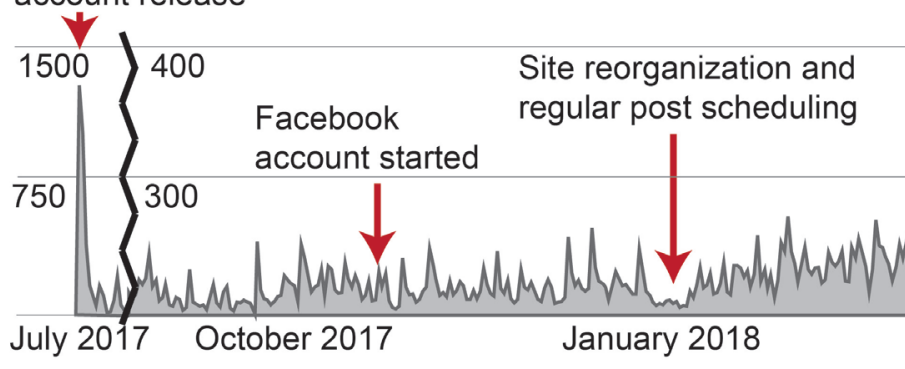

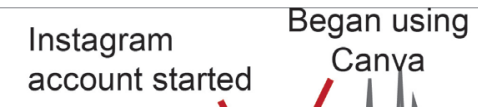

account started Canya

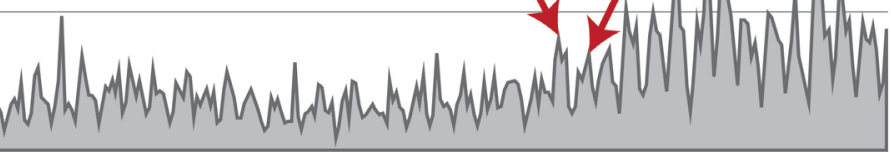

April 2018

July 2018

October 2018 
posts, write text, and then schedule a time and date for post releases.

Currently, none of our site collaborators or curators are in permanent positions, so our time contributed towards the site is outside of our dissertations, theses, postdoctoral and visiting professor teaching and research responsibilities. Funds required to keep the site operational have been acquired through several small grant programs and gifts from universities. Additional funding is acquired through Patreon.com, a platform for creators that allows supporters to contribute to and support their work. Currently, Time Scavengers has monthly contributors who gain access to new content early in return for their continued financial support of the website.

\section{SITE NAVIGATION}

The Time Scavengers website comprises both dynamic blogs and informational page components (Figure 1). Informational pages include the 'Home' page, with the site's objectives and information on how best to navigate through the content; an 'About Us' section with information about the site collaborators, a project overview, and contact information. The 'Introductory Material', 'Climate Change', 'Evolution', 'Educational Resources', and 'Extra Information' pages contain the bulk of the educational static pages. Many of these pages provide additional subpages to break down the complex content into smaller concepts, with the goal of the reader learning as they progress through the site. Additionally, the site hosts six blog components: 'Field Excursions', 'Education \& Outreach', 'Science Bytes', 'Byte of Life', 'Meet the Scientist', and 'Climate \& Paleo News'.

Content added to the site that required additional research or came directly from a scientific reference includes citation information. This information was then linked to our comprehensive 'References' page. The goal of linking references from the primary literature is to provide reliable information to the public, and especially educators, who may want to use the information in talks, lectures, or other teaching/outreach opportunities. Including sources on the pages makes the data presented more credible, which has been found to play a major role in a blog's success and leads to a positive reader's perception of the material (Colliander and Dahlen, 2011). Each of the informational pages and blogs add to the depth of content on climate change and evolutionary theory and helps explain the sometimes labyrinthine process of scientific endeavors.

Informational Pages. The 'Home' page of Time Scavengers contains an overview of the site, the main objectives of the material posted, and a mission statement. In addition to the 'Biography' page, the two site curators, ARL and JEB, who are the main bloggers on the site, each have a page to more fully expand on their research interests through the
'Our Research Explained' pages (Figure 1). As blogs can provide a connection among readers and bloggers, the expanded research and biography pages act as avenues through which site visitors can better know the scientists blogging, thus establishing a feeling of friendship and trust (Colliander and Dahlen, 2011).

The spread of complexity of content throughout the informational pages allows for formal and informal learners to decide how they need to navigate through the site. Informal learners with less scientific training may need to start on the 'General Science' page, which walks the user through the scientific method, hypothesis testing, and how to think about biases within data. Within these sections are pages that cover the most common sedimentary rocks containing fossils (used in both evolutionary and climate change research) and common shell and skeleton forming minerals. The 'Introductory Material' section of the site also includes a page about 'Geologic Time', which explains how the geologic time scale was developed and the common methods used in geochronology. Two significant subsections under the 'Introductory Material' are 'What is Paleontology?' and 'What is Paleoclimatology?', pages that explicitly explain these two disciplines. Each subsection contains additional pages explaining topics crucial for interpreting climate and evolutionary data, such as 'Types of Data' and 'Proxy Data'. Once users have read all or part of the introductory pages, or for those more versed in these topics, users can then move to the 'Climate Change' and 'Evolution' sections of the site.

Two major sections of the site, 'Climate Change' and 'Evolution', contain themed pages with respect to each topic (e.g., 'Ocean Circulation', 'Taxonomy') that more fully explain the science behind each (Figure 1). The pages are designed so that the content builds from one page to another, much like a lecture-based course or classroom environment. This way, as readers progress, they can apply what they learned on the previous page as they move to the next. Each of the static informational pages possess simple figures drafted or modified from pre-existing work by the Time Scavengers team to ensure simplicity. Most scientific figures, charts, and graphs are incredibly complex and multifaceted, which is a factor that often deters the public from accepting and learning about climate science (Weber and Stern, 2011).

Blogs. In addition to the informational pages, the site contains six blog components (Figure 1). Four of the blogs ('Field Excursions', 'Education \& Outreach', 'Science Bytes', and 'Byte of Life') are housed under the heading of 'Paleo LIVE!', an area of the site made for the blogs that are specifically about data collection, research, outreach, and lives our site collaborators lead. The other two blogs, 'Meet the Scientist' and 'Climate \& Paleo News', are designed to introduce the public to different scientists and disseminate the major findings and implications of recent publications 
in the field of climate science and evolutionary theory. Research into factors that make people more open to digesting climate change data (e.g., O'Neill and Nicholson-Cole, 2009) has indicated that sharing of personal stories and experiences is more effective at reaching and influencing the public than scare tactics and lambasting with climate facts. The blogs serve to do just that: share personal stories and experiences related to climate change and evolutionary science to engage and interest the public in these topics.

All the blogs are written by different members of the Time Scavengers team, whose rich backgrounds, identities, and experiences aid in opening our lives as scientists to the public. For example, 'Science Byte' is a blog where we explain what the data are that we use in our own research, where they come from, and how we interpret them. The site showcases a diverse group of scientists and their science on our 'Meet the Scientist' blog, with the hope of increasing visibility of scientists who belong to historically marginalized groups (including but not limited to LGBTQ+ individuals, people of color, disabled persons, and women) and exposing young science enthusiasts to different fields of study. We also recognize that not all scientists obtain doctorate degrees, thus we work to feature non-traditional scientists to highlight that scientific discovery is not limited to those with advanced degrees. The main purpose of the Time Scavengers blog series is to continue to provide advice, support, ideas, and information to the public, our peers, and learners.

Educational Resources and Extra Information. Time Scavengers caters to K-12 and college/university educators by curating a page dedicated to educational resources related to paleontology, evolution, paleoceanography, modern climate, and climate change, among other topics. Here, classroom activities are listed and separated by general categories. Each activity has a link to the original resource or website as well as a short description about the activity and for what grade level it is appropriate. In addition to educational materials, we also include other paleontology-related activities and resources. For example, we recently compiled a list of paleontology-related summer camps and provide a link to the camps at the top of the 'Educational Resources' page. This was a request from a community member on Twitter and we made the list publicly available. Other related resources, whether requested from our followers or not, will continue to be added to this page in the future.

Time Scavengers also hosts a series of pages for visitors who want to expand their knowledge on any geoscience topic, and to further support educators and the general public with interests in the natural sciences. The 'Extra Information' section contains four subsections, including 'Additional Reading', 'Organizations \& Societies', 'Fossil Identification \& Societies', and 'Podcasts \& Blogs'. Here, users can find lists of books (separated by topic), a list of organizations and societies (both professional and avocational) across the United States, fossil identification databases and websites, and paleo-related blogs and podcasts. All of these pages are continually being updated with new resources.

\section{DATA COLLECTION AND OUTREACH ASSESSMENT}

The primary goal of Time Scavengers is to disseminate accurate scientific information to the public. To ensure that this goal is being met as efficiently as possible, we have been collecting data on the site's traffic through Google Analytics since the site's public release in July 2017. Some examples of data collected include demographics and location of users; length of time users stay on a page; number of new versus returning users; information that visitors to the site are clicking on; type of link that led users to the site; and the way that users move through the website. Through Analytics we can also determine how visitors are reaching the site, either through our social media platforms (Facebook, Twitter, and Instagram), or through search engines and keywords (Figure 3). These statistics and metrics help us learn how we can best disseminate information to the public and make the site more effective. For example, when we first assessed the site statistics in October 2017, our user base was 93\% English-speaking (Bauer et al., 2017). We then examined the percentages of users who visited the site with a web browser (e.g., Chrome, Firefox, Safari) that had automatic translation capabilities and those that did not. Because many of the web browsers visitors were using did not automatically translate text into their native language, we installed a Goo-

Figure 1. Data on how people are reaching the Time Scavengers website. Organic searches are those visitors who find Time Scavengers through internet search engines using specific key words; referrals are when people reach the site through ads and links posted on other websites and pages; direct traffic comes from visitors entering a Time Scavengers URL into their web browser or using a bookmark to access the website; and social media traffic comes from people clicking a link on a social media platform. Within the social media category, most traffic finds the site through Facebook. The 'other' category includes the social media sites TypePad, LinkedIn, Blogger, YouTube, Instagram Stories, and WordPress.

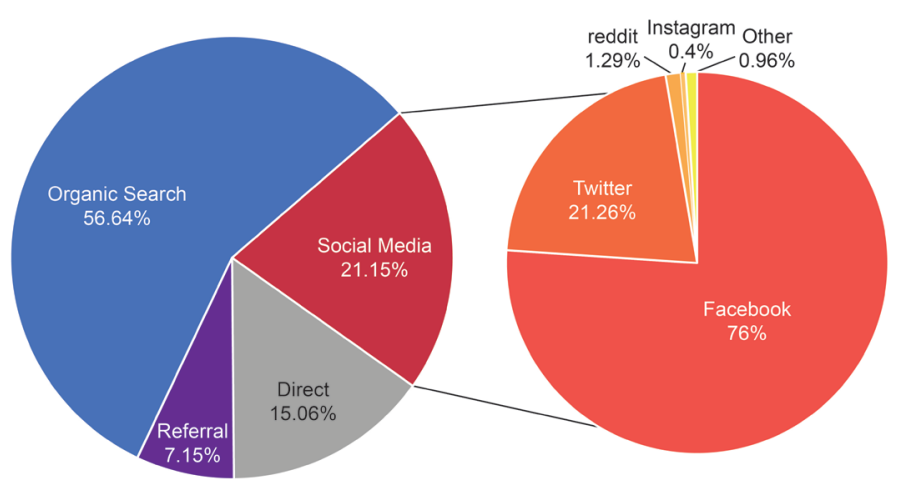


gle Translate plugin that translates the site into the user's preferred language regardless of browser used. Two months later, in December 2017, the percentage of English-speaking users had decreased from $93 \%$ to $88 \%$ (Lam et al., 2017). To attract more international visitors to the site, we are inviting non-U.S. scientists to write about themselves and their science in their native language on our 'Meet the Scientist' blog.

Additionally, our social media accounts collect all user interactions associated with a post. Each post can be assessed by views, clicks, shares, likes, comments, and more (Figure 4). These data help us determine what type of content performs best on which social media platforms.

\section{STRATEGIES FOR REACHING THE PUBLIC AND BUILDING AN AUDIENCE}

The challenge of any website or blog created for the public is gaining both an audience and a reliable following. Time Scavengers has created an online community and uses a variety of tools to expand its reach to sustain and grow its audience. The site's social media accounts are utilized to promote the blog posts and having positive interactions with our community, such as answering questions related to our content or sharing fossil images. We publish additional posts related to a new fossil each week, resources and articles related to climate change and evolutionary theory, educational materials for classroom use, and to promote our informational pages.

Proficiency at utilizing social media as a tool to reach a broader audience includes using hashtags relevant to the post to help others outside of the Time Scavengers community find our content. We now regularly participate in weekly hashtags, especially \#FossilFriday. This tactic has been incredibly beneficial in expanding our audience, particularly on Instagram and Twitter, as an increased number of users who do not follow Time Scavengers often engage with our posts by liking, sharing, or commenting on the post of interest (Figure 4). Days that include social media posts correspond to spikes in daily active users (Figure 2C; Figure 4C).

\section{FUTURE DIRECTIONS}

As the Time Scavengers community grows, so will the site and its contents. Immediate plans for the site include adding more informational pages related to general science,

Figure 1. Example of a Facebook and Twitter post promoting our 'Isotopes' informational page, with data on each of the posts' social media interactions. (A) The 'Isotopes' post on Facebook (left) and Twitter (right). (B) Post performance comparison of both Facebook and Twitter posts. Facebook provides a detailed summary of public interactions with the post. Twitter provides information similar to Facebook, but also indicates how many times users have clicked on the Time Scavengers profile. (C) Google Analytics summary of number of site visitors from July 1st, 2018 to September 1st, 2018. On the date this post was released (August 28th, 2018, indicated by the red arrow), the Time Scavengers site had 26\% returning visitors and $76 \%$ new visitors to the site.

A

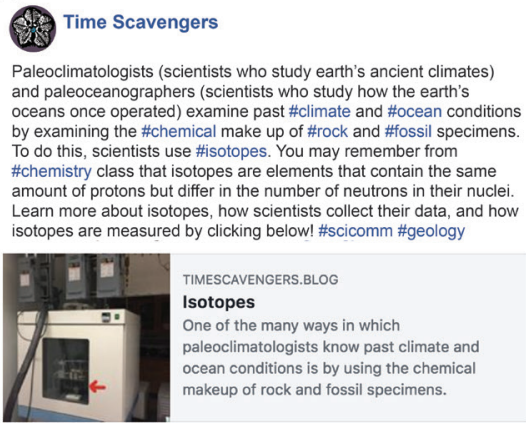

C

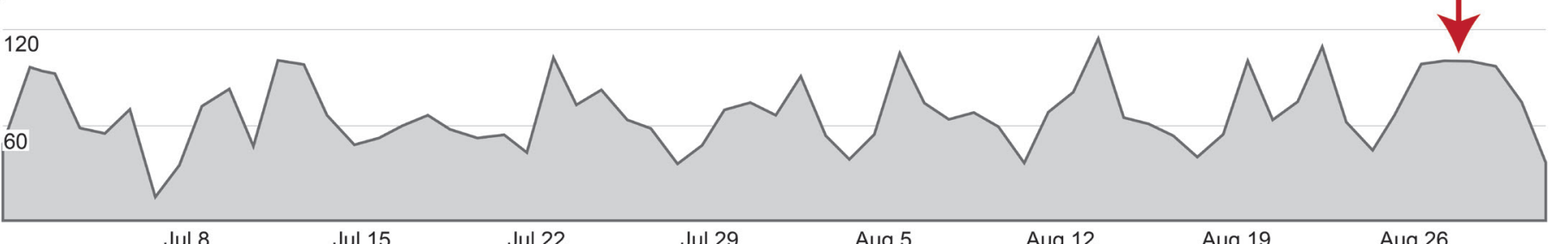

Post performance

\begin{tabular}{l|c|c} 
& Facebook & Twitter \\
\hline Reach & 504 & 1,913 \\
\hline Likes & 15 & 8 \\
\hline Shares/Retweet & 4 & 5 \\
\hline Post clicks & 26 & 3 \\
\hline Link clicks & 6 & 11 \\
\hline Profile clicks & - & 5
\end{tabular}

August 28

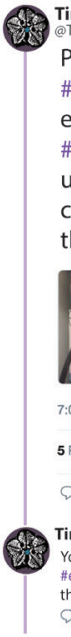
You may remember from \#chemistry class that isotopes are the number of neutrons in their nuclei. \#scicomm \#geology the number of neutrons in their nuclei. \#scicomm \#geology

Paleoclimatologists examine past examining the \#chemical make up of \#rocks and \#fossils. To do this, scientists use \#isotopes. Learn more about how to collect \& measure isotopes by clicking the link!

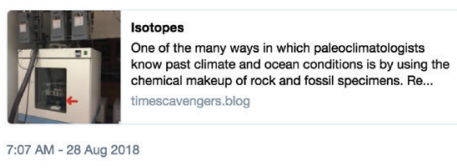

Retweets 8Likes

7750

$\gamma_{5}$
B ons in their nucl

up of rock and fossil specimens.

Jul 8

Jul 15

Jul 22

Jul 29

Aug 5

Aug 12

Aug 19

Aug 26 
climate change, and evolution. Some future pages will include 'Plate Tectonics', 'Paleobiogeography', and 'Continental Ice Sheets'. Additionally, we aim to reach a more global following by increasing the number of international scientists featured on our 'Meet the Scientist' blog. Regarding educational activities and resources for educators, the 'Educational Resources' page will continue to grow as new activities relating to climate change and evolution are added. We also plan to develop and modify our own educational activities based on the information contained on Time Scavengers. Students could explore advanced topics that are explained thoroughly through the site and then apply their knowledge using published or publicly-available datasets. By using pre-existing databases such as iDigBio (www. idigbio.org) or Climate Data Online (www.ncdc.noaa.gov), learners can explore human impact on the climate, how diversity has changed through time, and much more.

To accommodate site visitors who may be more visual learners, we will curate and expand a YouTube channel and include more blog posts that are image-heavy. Recent work by Sheffield et al. (2018) examined the engagement rate of posts with many appealing photos accompanying text compared to posts with fewer photos and found that readers engage more with posts that have a higher number of quality images. Thus, future blog posts will feature a higher image to text ratio. In attempt to reach the underrepresented age groups (Fig. 2B), we will share related posts into more groups on Facebook and use the advertising features on these platforms to target more specific groups of people. We can then analyze the results of these ads to see if our users increase in specific age brackets (Lam and Bauer, 2018; Bauer et al., in prep).

\section{IMPACT}

Time Scavengers has grown into a hub for climate change- and evolution-related informational pages, resources, and blogs. The informational pages and blogs are written as non-technical and free from scientific jargon as possible, making the site an ideal resource for middle school through university students and beyond who want to learn more about the geosciences. Because the informational pages contain links to sites for further research, the site is also ideal for community scientists and informal learners who wish to learn more about the world around them. Currently, our Google Analytics data indicates that $70.9 \%$ of site users are from the U.S., with the other $29.1 \%$ of visitors from 129 other countries. Time Scavengers is reaching a global audience and will continue to broaden its reach in the future.

\section{AUTHOR INFORMATION \\ Corresponding Author}

Adriane Lam,arlam@geo.umass.edu

\section{Author Contributions}

The manuscript was written through contributions of all authors. All authors have given approval to the final version of the manuscript.

\section{FUNDING SOURCES}

Funding for Time Scavengers is from a Paleontological Society Outreach and Education Grant to ARL and JEB, the Department of Earth and Planetary Sciences at the University of Tennessee Knoxville, a year of site support from WordPress, and crowdsourced funding through Patreon (www. patreon.com/TimeScavengers).

\section{ACKNOWLEDGMENTS}

ARL and JEB would like to acknowledge the unwavering support of this project by their current and former advisors AL Stigall, RM Leckie, and CD Sumrall. We would like to thank two anonymous reviewers for their constructive comments that greatly improved this manuscript.

\section{ABBREVIATIONS}

ARL: Adriane R. Lam; JEB: Jennifer E. Bauer; SF: Susanna Fraass.

\section{REFERENCES}

Bauer, J.E., Lam, A.R., Bryant, R., Fraass, A. J., Golder, K., Hartshorn, K. R., Hils, J. M., Limbeck, M., and Sheffield, S. L. (2017). Time Scavengers: A collaborative website for exploring climate change and evolution through scientists' experiences. Geological Society of America Meeting, Abstracts with Programs, 49(6), doi: 10.1130/ abs/2017AM-295553

Colliander, J., and Dahlén, M. (2011). Following the fashionable friend: The power of social media: Weighing publicity effectiveness of blogs versus online magazines. Journal of Advertising Research, 51(1), 313-320.

Hanna, R., Rohm, A., and Crittenden, V. L. (2011). We're all connected: The power of social media ecosystems. Business Horizons, 54, 265-273.

Lam, A.R., Bauer, J.E., Bryant, R., Fraass, A.J., Golder, K., Hartshorn, K.R., Hils, J.M., Limbeck, M., and Sheffield, S.L. (2017). Time Scavengers: A website for the public to learn about climate change and evolution through the experiences of scientists. American Geophysical Union Fall Meeting, Louisiana, USA.

Lam, A.R., and Bauer, J.E. (2018). Using social media paid advertisements, sharing, and engaging posts to reach a wider audience and increase climate change and evolu- 
tionary theory literacy. Geological Society of America Meeting, Abstracts with Programs, 50(6), doi: 10.1130/ abs/2018AM-319985

Lazer, D.M., Baum, M.A., Benkler, Y., Berinsky, A.J., Greenhill, K.M., Menczer, F., Metzger, M.J., Nyhan, B., Pennycook, G., Rothschild, D., Schudson, M., Sloman, S.A., Sunstein, C.R., Thorson, E.A., Watts, D.J., and Zittrain, J.L. (2018). The science of fake news: Addressing fake news requires a multidisciplinary effort. Science, 359(6380), 1094-1096. doi:10.1126/science.aao2998

National Center for Science and Engineering Statistics, National Science Foundation. (2016). Survey of Earned Doctorates 2016. Retrieved from https://www.nsf.gov/statistics/2018/ nsf18304/

O'Neill, S., and Nicholson-Cole, S. (2009). Fear won't do it: Promoting positive engagement with climate change through visual and Iconic Representations. Science Communication 30(3), 355-379.

Sheffield, S.L., Bauer, J.E, and Lam, A.R. (2018). Making geology accessible through engaging blogs: Using photos to bring the outdoors in. Geological Society of America Meeting, Abstracts with Programs, 50(6), doi: 10.1130/ abs/2018AM-321664

United States Census Bureau. (2016). 2014 National population Projections Dataset. Retrieved from https://www.census. gov/data/datasets/2014/demo/popproj/2014-popproj.html.

Weber, E. U., and Stern, P. C. (2011). Public understanding of climate change in the United States. American Psychologist 66(4), 315-328.

Wilkins, J. S. (2008). The roles, reasons, and restrictions of science blogs. Trends in Ecology \& Evolution 23(8), 411-413. 